\title{
Circuit
}

Musiques contemporaines

\section{Introduction. Réflexions sur le métier de compositeur : identité et singularités}

\section{Simon Bertrand}

Volume 27, numéro 1, 2017

Réflexions sur le métier de compositeur : identité et singularités

URI : https://id.erudit.org/iderudit/1039668ar

DOI : https://doi.org/10.7202/1039668ar

Aller au sommaire du numéro

Éditeur(s)

Les Presses de l’Université de Montréal

ISSN

1183-1693 (imprimé)

1488-9692 (numérique)

Découvrir la revue

Citer ce document

Bertrand, S. (2017). Introduction. Réflexions sur le métier de compositeur :

identité et singularités. Circuit, 27(1), 9-11. https://doi.org/10.7202/1039668ar d'utilisation que vous pouvez consulter en ligne.

https://apropos.erudit.org/fr/usagers/politique-dutilisation/ 


\section{Introduction. Réflexions sur le métier de compositeur : identité et singularités}

Simon Bertrand

Par le biais de divers portraits, enquêtes, analyses d'œuvres et textes d'opinion mettant en relief les démarches artistiques ou sociales de compositeurs et de compositrices représentant plusieurs générations, ce numéro de Circuit porte un regard sur le métier de compositeur dans le contexte québécois en ce début de XXI ${ }^{\mathrm{e}}$ siècle, et aborde en filigrane les questions de l'identité et de la singularité, l'exercice de cette profession et notamment selon le milieu où elle est exercée. Ce numéro souhaite aussi générer, par la même occasion, des réflexions et des questionnements d'ordre sociologique sur le milieu de la création musicale au Québec et ses racines; celles, encore fragiles, du passé, et celles qu'il reste à inventer.

Dans l'histoire de la musique, il y a toujours eu des démarches compositionnelles plus difficiles à associer que d'autres à un courant particulier. Le $\mathrm{xx}^{\mathrm{e}}$ siècle musical a eu, comme tous les autres, sa part d'iconoclastes, d'inclassables qui nous ont souvent rappelé à quel point la recherche d'une esthétique musicale singulière est quelque chose d'intime. Celle-ci n'est donc pas nécessairement toujours rattachée de manière ombilicale à de grands courants ou écoles de pensées, ces catégorisations étant souvent le fruit d'une musicologie historiographique ou alors d'une autoaffiliation volontaire et délibérée des compositeurs eux-mêmes tel que cela fut souvent le cas au $\mathrm{Xx}^{\mathrm{e}}$ siècle.

Il suffit de penser à quelques cas de compositeurs qui, s'ils ont indéniablement marqué l'évolution du langage de la musique de concert au $\mathrm{XX}^{\mathrm{e}}$ siècle, n'ont jamais adhéré à un système ou à un concept compositionnel précis: Charles Ives, Luciano Berio, ou d'autres qui ne sont jamais restés figés dans une de leurs «manières ", György Ligeti en étant l'un des exemples les plus éloquents. Il y a aussi d'autres compositeurs qui, véritables forces tranquilles, ont fait leur chemin en solitaire, en toute singularité, sans adhérer à un 
1. André Mathieu (1929-1968) est un pianiste et compositeur québécois. Sa musique, notamment son Concerto $n^{\circ} 3$ pour piano et orchestre, connut sous le nom de Concerto de Québec un grand succès public grâce notamment aux concerts et enregistrements du pianiste Alain Lefèvre, essentiellement entre les années 2005 à 2010. Paru en 2005 chez Analekta, son disque Hommage à André Mathieu a été vendu à plus de 40000 exemplaires.

2. "Un "présent perpétuel" où l'acte de création n'est pas motivé tant par le progrès, l'historicisme, le courant, l'idéologie et j'en passe, que par la création dans le désir de s'exprimer, de contribuer, de durer au quotidien." Voir ici-même l'article de Danick Trottier. courant ou à une quelconque mode, et sont restés relativement fidèles à une certaine démarche esthétique. Je pense ici notamment à Benjamin Britten, à Henri Dutilleux, Maurice Ohana (encore trop méconnu), à l'Américain Elliot Carter, ou encore, au Québec, à José Evangelista, à Bruce Mather et, évidemment, à Claude Vivier.

D’une certaine manière, les quatre compositeurs auxquels ce numéro de Circuit s'attarde un peu plus spécifiquement - Serge Arcuri, Julien Bilodeau, Linda Bouchard et André Hamel - sont des exemples d'une ou l'autre de ces formes de singularité.

Cependant, il ne faut pas oublier que c'est une émulation de certains canons plus établis de la musique d'avant-garde européenne qui fut à la base de la constitution même du milieu, au Québec, de la musique nouvelle. Mais celui-ci est-il réellement «né » subitement, un beau matin des années 1960, en pleine Révolution tranquille, en une sorte de reflet ce qui se faisait dans les hauts lieux de la musique d'avant-garde en France et en Allemagne, comme à Darmstadt ou au Domaine Musical? Et cette émulation de l'Europe futelle nécessairement synonyme d'une absence ou d'un renoncement à toute «américanitude », passée ou à venir, de notre création musicale?

Au sujet de la question des racines, si l'auteur de ces lignes avoue qu'il aurait personnellement préféré que le très médiatisé pianiste québécois Alain Lefèvre mette son talent et son «star power » au service de la musique du compositeur Rodolphe Mathieu plutôt que de celle de son fils André Mathieu ${ }^{1}$ et son esthétique certes un peu décalée pour l'époque, le "phénomène Mathieu » a quand même, je crois, amené le milieu de la création musicale à réfléchir sur ses racines. Si l'on faisait l'exercice de demander à des passants dans la rue de citer les noms de grands écrivains, poètes, cinéastes ou peintres québécois, on obtiendrait sans doute quelques incontournables, comme Anne Hébert, Émile Nelligan, Denys Arcand, Jean-Paul Riopelle. On risquerait par contre d'être confronté à un silence de mort en demandant le nom ne fusse que d'un grand compositeur québécois de musique de concert, à moins qu'avec un peu de chance on obtienne le nom, justement, d'André Mathieu...

Larticle de Danick Trottier aborde la question du «présentisme²». Larticle tente de démontrer que les déchirements au sein du modernisme entre un héritage lourd à porter et le besoin de faire progresser le langage musical a aujourd'hui cédé le pas à une dialectique plus subtile où s'agglomèrent les catégories temporelles du passé, du présent et du futur, le passé devenant un héritage plus ouvert, mais interprété dans les limites et les enjeux du présent. 
Londiste et compositrice Estelle Lemire dresse un portrait intimiste du compositeur Serge Arcuri tandis que je me suis entretenu avec la compositrice québécoise «exilée» aux États-Unis Linda Bouchard. Deux véritables forces tranquilles qui ont construit leur carrière de compositeur indépendamment des institutions d'enseignement, en véritables artisans, se consacrant exclusivement à la composition musicale et possédant une démarche artistique très personnelle, que l'on pourrait difficilement assimiler à un seul courant esthétique.

Depuis une dizaine d'années, un type d'initiative moins fréquent dans le passé semble s'être développé: celui des résidences de compositeurs au sein d'ensembles, d'orchestres ou d'organismes de diffusion. Une enquête portant sur ce défi particulier - qui consiste à mettre le compositeur dans un cadre où il peut à la fois se développer artistiquement et apprendre à communiquer avec le public afin d'expliquer sa démarche et vulgariser son métier - me semblait donc s'imposer. Trois compositeurs - Cléo Palacio-Quintin, Nicolas Gilbert et Tim Brady - ayant effectué ou effectuant présentement de telles résidences ont généreusement accepté de se prêter au jeu.

Le Cahier d’analyse, signé Paul Bazin, porte sur Urnos, grande mystification d'un esprit indépendant et iconoclaste à sa manière, André Hamel, qui contribue au questionnement de la singularité et de l'identité avec une œuvre jouant, selon Bazin, sur une «notion de musique élémentielle qui lui est propre et qui confère au corpus de ses œuvres récentes une part de leur singularité».

Au sujet des racines réelles, imaginaires ou manquantes de la création musicale au Québec, et surtout de la notion de marge, le compositeur Marc Hyland nous offre dans la chronique «Entendu dans Cette ville étrange» une réflexion très personnelle qui pourra peut-être s'ajouter à celles des articles «"Fuck toute!" - une génération de compositeur.e.s décomplexe sa marge ${ }^{3}$ ” de Symon Henry et «D'accessibilité et d'authenticité4 » d'André Hamel, tous deux publiés dans cette même chronique de numéros antérieurs de Circuit.

Ce numéro, par ramification, amènera sans doute bien plus de questionnements, notamment sur la place des créateurs de musique dans une culture globale que de quelconques certitudes musicologiques, esthétiques ou historiques. Si c'est le cas, il aura alors atteint ses objectifs.

Bonne lecture!

Montréal, février 2017

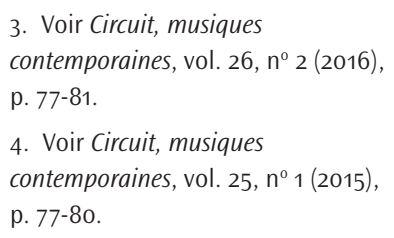

